\title{
On the non-Boltzmannian nature of quasi-stationary states in long-range interacting systems
}

\author{
Constantino Tsallis \\ Centro Brasileiro de Pesquisas Fisicas, Rua Xavier Sigaud 150, 22290-180 Rio de \\ Janeiro-RJ, Brazil \\ Andrea Rapisarda \\ Dipartimento di Fisica e Astronomia, Universitá di Catania, \\ and INFN sezione di Catania, Via S. Sofia 64, I-95123 Catania, Italy \\ Alessandro Pluchino \\ Dipartimento di Fisica e Astronomia, Universitá di Catania, \\ and INFN sezione di Catania, Via S. Sofia 64, I-95123 Catania, Italy \\ Ernesto P. Borges \\ Escola Politecnica, Universidade Federal da Bahia, Rua Aristides Novis 2, \\ 40210-630 Salvador-BA, Brazil
}

\begin{abstract}
We discuss the non-Boltzmannian nature of quasi-stationary states in the Hamiltonian Mean Field (HMF) model, a paradigmatic model for long-range interacting classical many-body systems. We present a theorem excluding the Boltzmann-Gibbs exponential weight in Gibbs $\Gamma$-space of microscopic configurations, and comment a paper recently published by Baldovin and Orlandini (2006). On the basis of the points here discussed, the ongoing debate on the possible application, within appropriate limits, of the generalized $q$-statistics to long-range Hamiltonian systems remains open.
\end{abstract}

Key words: Metastability in Hamiltonian dynamics; Long-range interactions; Nonextensive statistical mechanics.

Email addresses: tsallis@cbpf.br (Constantino Tsallis), 


\section{Introduction}

The Hamiltonian Mean Field (HMF) model is a paradigmatic toy model for long-range interacting Hamiltonian systems [1]. In a recent paper [2], Baldovin and Orlandini presented new molecular dynamics numerical results for the HMF model in contact with a thermal bath. They conclude that the energy distribution in its quasi-stationary state (QSS) is of the Boltzmann-Gibbs (BG) form. In view of their own numerical results, we cannot agree with their conclusions. In this paper we present three points that are relevant for the interesting questions raised in [2].

\section{Discussion}

In what follows we present three important points regarding the non-Boltzmannian nature of the QSS in the HMF model.

(i) The nature of the QSS in the canonical setup presents differences with that in the microcanonical setup.

It has already been demonstrated that, in the microcanonical ensemble, the anomalous dynamics (concerning the presence of a glassy phase, hierarchical structures, velocity correlations, aging, vanishing Lyapunov exponents, etc), and therefore the statistical description of the QSS, is strongly dependent on the initial conditions [3,4,5]. A fortiori, it is thus not at all justified to extrapolate the conclusions for the canonical QSS studied in [2,6] to the microcanonical ones. Although the authors themselves state in [6] that the canonical QSS's are only reminiscent of the microcanonical ones (in fact they differ in the lifetimes, the relaxation times, and, most probably, also in the correlations), they neglect it in [2]. In order to stress this point, in Fig. 1 (a) we plot three numerical simulations in the microcanonical ensemble (averaged over 10 events) for $U=0.69, N=5000$ and for different initial magnetizations $M_{0}$. Water-bag initial conditions were used [3]. Following the Central Limit Theorem aspects recently revealed in [7], we illustrate in Fig. 1 (b) the non-Boltzmannian nature of this QSS regime by studying the probability density functions (pdfs) of the stochastic values $y$ defined as follows: $y_{j}=\sum_{i=1}^{n} l_{j}(i)$, for $j=1,2, \ldots, L$, where $l_{j}(i)$ are the momenta of the $j t h$-rotator taken at fixed intervals of time

andrea.rapisarda@ct.infn.it (Andrea Rapisarda), alessandro.pluchino@ct.infn.it (Alessandro Pluchino), ernesto@ufba.br (Ernesto P. Borges). 

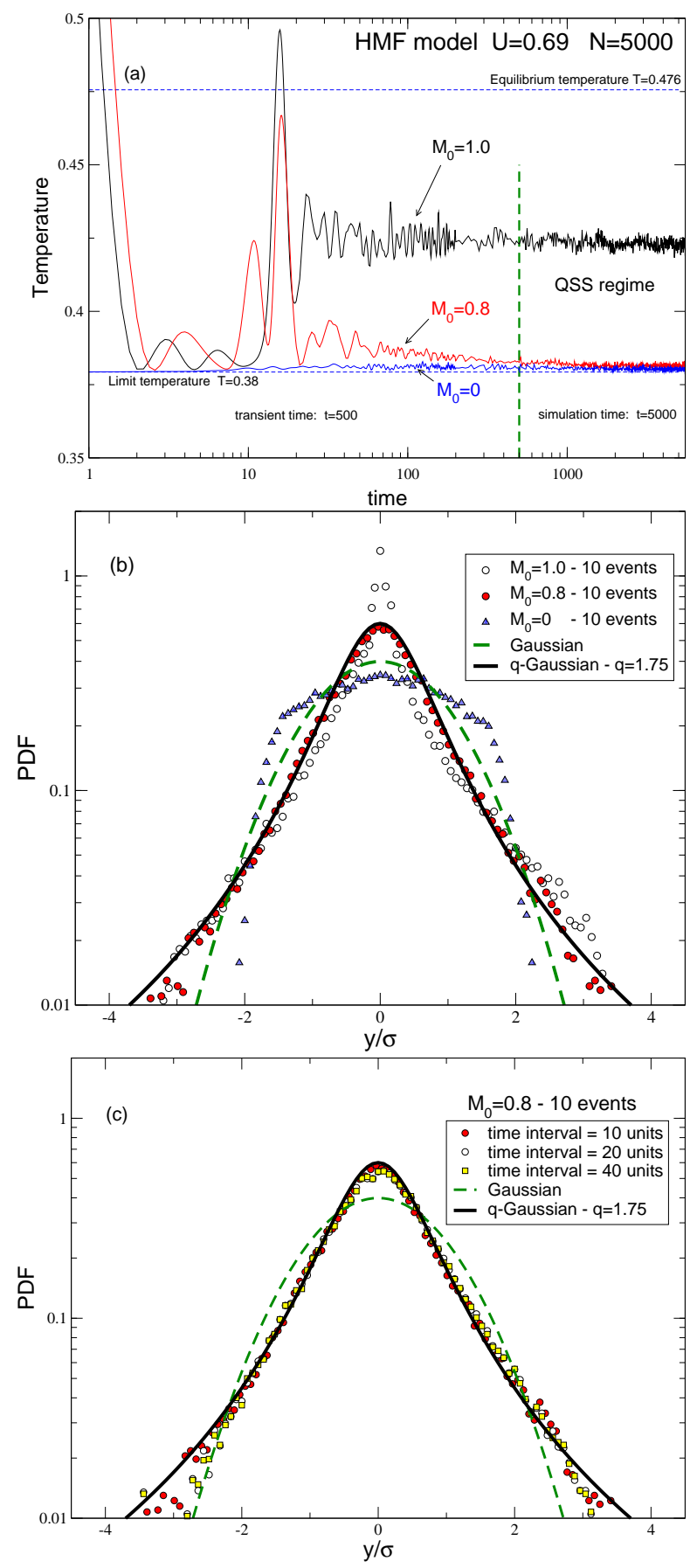

Fig. 1. (a) The temperature as a function of time for the HMF model at $U=0.69$ and $N=5000$ obtained for three initial magnetization values, respectively $M_{0}=1,0.8,0$, and averaged over 10 events. The system remains trapped in a QSS at a temperature value lower than the equilibrium one. The latter is drawn together with the limit temperature at this energy [3]. (b) The pdf of stochastic values $y$ obtained by summing rotators momenta in the QSS regime illustrated in panel (a), see text. Different initial magnetizations $M_{0}$ are reported. A Gaussian (dashed curve) and a $q$-Gaussian with $q=1.75$ (full curve) are also reported for comparison. (c) For the initial magnetization $M_{0}=0.8$, we plot the pdfs obtained changing the time interval for recording the momenta of the rotators. No significant change is observed. See text for further details. 
$i$ inside the QSS regime. We considered $L=5000$ and $\mathrm{n}=500$ intervals, one every 10 units of time. The length of the time interval was varied as shown in Fig. 1 (c) for the initial magnetization $M_{0}=0.8$ without any relevant change in the pdfs. An average over 10 events was also considered, in order to have 50000 values for the $y$ variables for each pdf. In case of no global correlations, as one would expect at equilibrium, the resulting pdf should be a Gaussian. As Fig. 1(b) shows, in our case, the Gaussian distribution does not occur. In fact we have pdfs whose shape depends on the initial magnetization $M_{0}$ and the central region (up to a few mean standard deviations) of most of them is well fitted by $q$-Gaussians. In particular for $M_{0}=0.8$ we get a $q$-Gaussian with $q=1.75$ as in [7]. We do not think that this is a universal value in this case, since we do not have a strictly zero largest Lyapunov exponent as in [7. For the HMF model, the latter goes to zero as $1 / N^{1 / 9}$ in the QSS regime [4], while it remains finite at equilibrium at this energy value. We normalized the data to the corresponding standard deviations in order to compare them. This comparison clearly shows that strong correlations are present in our case indicating a deviation from the standard Central Limit Theorem, as in [7]. A more complete study on this point will be reported elsewhere. In any case the arguments discussed in [2], and in particular in Fig. 1(a) of [2], do not apply to the microcanonical QSS. The effects become increasingly stronger when the initial magnetization gets closer and closer to 1.

(ii) The energy distribution of the QSS cannot be of the Boltzmann-Gibbs exponential form.

As clearly seen in Fig. 1(a) of [2], the one-body momentum distribution is nonGaussian. This excludes the BG exponential form as the energy distribution in full phase space $\Gamma$, as proved (by reduction to the absurd) in what follows. This analytical fact can easily be enlarged to $n$-dimensional rotators, $n>1$ : $n=1,2, d$, and $\infty$ correspond respectively to the Ising, $X Y$, Heisenberg, and spherical models. In what follows we illustrate it, however, for $n=2$, the case addressed in [2].

\section{Theorem}

Let $H=K+V$ be the Hamiltonian of a $M$-body classical system, where the kinetic energy is $K=\sum_{i=1}^{M} \frac{l_{i}^{2}}{2 I}, I>0$, and the potential energy $V$ contains arbitrary integrable one-body, two-body, three-body,... terms, concerning (isotropic or anisotropic) rotators localized at a (irregular or regular $d$-dimensional) lattice (each term might be characterized by a distance-dependent coupling constant, which can be summable or not in the $M \rightarrow \infty$ limit). And let the 
one-momentum marginal probability distribution

$$
p\left(l_{1}\right) \equiv \int d l_{2} d l_{3} \ldots d l_{M} d \theta_{1} d \theta_{2} \ldots d \theta_{M} p\left(l_{1}, l_{2}, \ldots, l_{M}, \theta_{1}, \theta_{2}, \ldots, \theta_{M}\right)
$$

associated with the joint distribution

$$
p\left(l_{1}, l_{2}, \ldots, l_{M}, \theta_{1}, \theta_{2}, \ldots, \theta_{M}\right)
$$

be non-Gaussian. Then

$$
p\left(l_{1}, l_{2}, \ldots, l_{M}, \theta_{1}, \theta_{2}, \ldots, \theta_{M}\right)
$$

cannot be proportional to

$$
e^{-\beta H\left(l_{1}, l_{2}, \ldots, l_{M}, \theta_{1}, \theta_{2}, \ldots, \theta_{M}\right)}, \beta>0
$$

\section{Proof:}

Assume that

$$
p\left(l_{1}, l_{2}, \ldots, l_{M}, \theta_{1}, \theta_{2}, \ldots, \theta_{M}\right)=\frac{e^{-\beta H}}{\int e^{-\beta H}},
$$

then

$$
\begin{gathered}
p\left(l_{1}\right)=\frac{\int d l_{2} d l_{3} \ldots d l_{M} d \theta_{1} d \theta_{2} \ldots d \theta_{M} e^{-\beta H}}{\int d l_{1} d l_{2} d l_{3} \ldots d l_{M} d \theta_{1} d \theta_{2} \ldots d \theta_{M} e^{-\beta H}}= \\
=\frac{\int d l_{2} d l_{3} \ldots d l_{M} e^{-\beta K}}{\int d l_{1} d l_{2} d l_{3} \ldots d l_{M} e^{-\beta K}} \frac{\int d \theta_{1} d \theta_{2} d \theta_{3} \ldots d \theta_{M} e^{-\beta V}}{\int d \theta_{1} d \theta_{2} d \theta_{3} \ldots d \theta_{M} e^{-\beta V}}=\frac{e^{-\beta l_{1}^{2} / 2 I \prod_{i=2}^{M} \int d l_{i} e^{-\beta l_{i}^{2} / 2 I}}}{\prod_{i=1}^{M} \int d l_{i} e^{-\beta l_{i}^{2} / 2 I}}= \\
=\frac{e^{-\beta l_{1}^{2} / 2 I}}{\int d l_{1} e^{-\beta l_{1}^{2} / 2 I}},
\end{gathered}
$$

which is a Gaussian, thus contradicting the hypothesis. Q.E.D.

Hence, the Central Limit Theorem verification exhibited in [2] refers to a necessary but insufficient property.

(iii) The Baldovin-Orlandini results do not exclude the nonextensive statistical mechanics q-exponential form.

Contrary to what is stated in [2], q-statistics [8] cannot be excluded on the basis of what they exhibit in their Fig. 3(d). Indeed, in Fig. 2 we plot the 


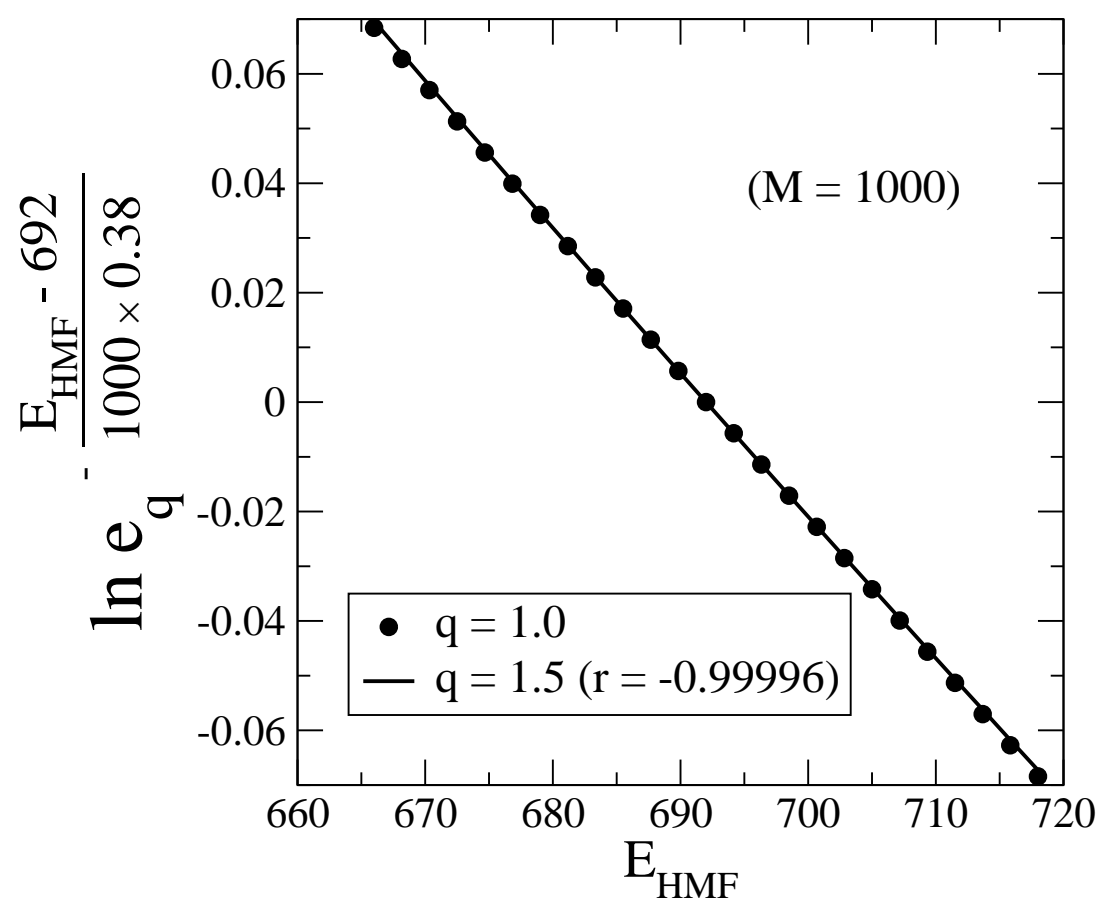

Fig. 2. The statistical weights corresponding to BG statistics (full circles), and to a typical illustration of nonextensive statistics ( $q=1.5$; solid line), for the $M=1000$ example exhibited in Fig. 3(d) of [2, see text for details.

statistical weights corresponding to BG statistics (full circles), and to a typical illustration of nonextensive statistics ( $q=1.5$; solid line), for the $M=1000$ example exhibited in Fig. 3(d) of [2]. We used nearly the same number of points of Fig. 3(d) in the interval $E_{H M F} \in[664,720]$, which covers $4 \%$ around its central value 692. The linear correlation is $r=-0.99996(r=-0.99997 \mathrm{in}$ [2] for a similar analysis). The remarkable closeness of the $q=1$ and $q=1.5$ examples comes from two facts. The first one is that

$$
e_{q}^{x} \equiv[1+(1-q) x]^{1 /(1-q)}=e^{x}\left[1+\frac{1}{2}(q-1) x^{2}+\ldots\right], \quad \text { for } x \rightarrow 0 .
$$

Notice the absence of the term linear in $x$ in the latter expression. While the second one is that the spreading around the central value 692 is very small. Notice also that we rescaled $T=0.38$ by a factor $M$, which corresponds to multiplying the Hamiltonian (1) of [2] by $\mathrm{M}$ and then rescaling time in the kinetic energy (see [9]). This makes the microscopic two-body interaction of the model independent from the macroscopic quantity $M$, as desirable. Consider also that, in the $q$-statistical weight of Fig. 2, we have subtracted from $E_{H M F}$ a characteristic macroscopic energy, namely the central value 692. This procedure is long known to be mandatory (see [10]) for any $q \neq 1$ in order to preserve the invariance of probabilities with regard to an uniform shift of the energy scale, i.e., in order to preserve the freedom of the choice of the zero of energies. Such subtraction, although allowed for all values of $q$, is clearly not mandatory for the BG exponential weight $(q=1)$, since the desired invariance 
is anyhow guaranteed by the fact that the exponential of a sum factorizes into the product of exponentials (whereas the $q$-exponential of a sum does not factorize into the product of $q$-exponentials if $q \neq 1$ ).

\section{Conclusions}

On the basis of the points here discussed, the results presented in [2] should be considered with extreme care, and by no means they close the ongoing debate on the possible application, in appropriate limits, of generalized $q$-statistics to long-range Hamiltonian systems. Finally, let us mention for completeness that neither the arguments recently advanced in [11] exclude the possible application of $q$-statistics, especially for initial magnetization close to 1 , but this point is beyond the scope of the present paper and it will be discussed elsewhere.

\section{Acknowledgments}

We acknowledge useful discussions with F. Baldovin. A.P. and A.R. acknowledge financial support from the PRIN05-MIUR project "Dynamics and Thermodynamics of Systems with Long-Range Interactions". C.T. acknowledges financial support from the Brazilian Agencies Pronex/MCT, CNPq and Faperj.

\section{References}

[1] T. Dauxois, V. Latora, A. Rapisarda, S. Ruffo and A. Torcini, in Dynamics and Thermodynamics of Systems with Long Range Interactions, T. Dauxois, S. Ruffo, E. Arimondo and M. Wilkens, eds., Lecture Notes in Physics 602 (Springer, Heidelberg, 2002) p. 458; A. Pluchino, V. Latora and A. Rapisarda, Continuum Mechanics and Thermodynamics, 16 (2004) 245; P.H. Chavanis, J. Vatteville and F. Bouchet, Eur. Phys. J. B 46 (2005) 61.

[2] F. Baldovin and E. Orlandini, Phys. Rev. Lett. 97 (2006) 100601.

[3] A. Rapisarda and A. Pluchino, Europhysics News 36 (2005) 202 and refs. therein; A. Pluchino and A. Rapisarda, Progress in Theoretical Physics Supplement 162 (2006) 18; A. Pluchino, V. Latora, and A. Rapisarda, Physica A 370 (2006) 573.

[4] V. Latora, A. Rapisarda, C. Tsallis, Physica A 305 (2002) 129; B.J.C. Cabral and C. Tsallis, Phys. Rev. E 66 (2002) 065101(R). 
[5] P.H. Chavanis, Eur. J. Phys. B 53 (2006) 487.

[6] F. Baldovin and E. Orlandini, Phys. Rev. Lett. 96 (2006) 240602.

[7] U. Tirnakli, C. Beck and C. Tsallis, Phys. Rev. E (2007), in press cond-mat/0701622.

[8] C. Tsallis, J. Stat. Phys. 52 (1988) 479; M. Gell-Mann and C. Tsallis, eds., Nonextensive Entropy - Interdisciplinary Applications (Oxford University Press, New York, 2004); J.P. Boon and C. Tsallis, eds., Europhys. News 36 (6) (2005).

[9] C. Anteneodo and C. Tsallis, Phys. Rev. Lett. 80 (1998) 5313.

[10] C. Tsallis, R.S. Mendes and A.R. Plastino, Physica A 261 (1998) 534.

[11] A. Antoniazzi, F. Califano, D. Fanelli, S. Ruffo, Phys. Rev. Lett. 98 (2007) 150202 and A. Antoniazzi, D. Fanelli, J. Barré, P.-H. Chavanis, T. Dauxois and S. Ruffo, Phys. Rev. E 75 (2007) 011112. 